\title{
Improved Yields with Added Copper(I) Salts in Carbonylative Stille Couplings of Sterically Hindered Vinylstannanes
}

\author{
R. D. Mazzola, Jr., Sören Giese, Chantel L. Benson and F. G. West* \\ Department of Chemistry, University of Utah, 315 S. 1400 East, Rm. Dock, Salt Lake City, UT \\ 84112-0850 and Department of Chemistry, University of Alberta, W5-67 Chemistry Centre, \\ Edmonton, AB T6G 2G2, Canada \\ frederick.west@ualberta.ca
}

\section{Contents:}

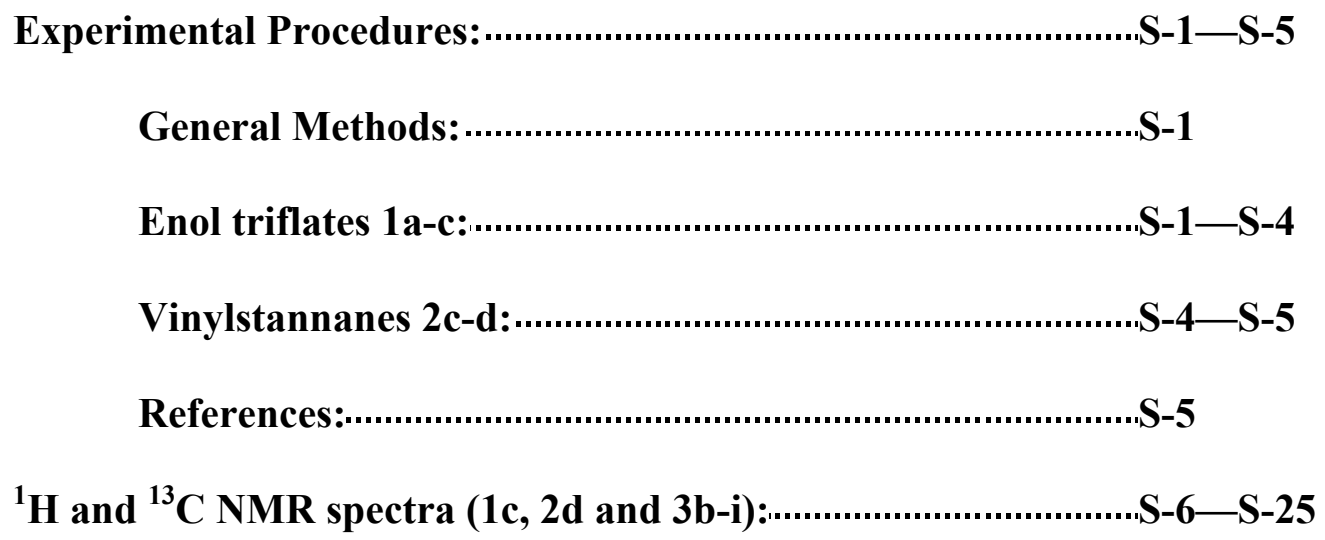


General Methods. Reactions were conducted in oven-dried $\left(120^{\circ} \mathrm{C}\right)$ or flame-dried glassware under a positive atmosphere of nitrogen unless otherwise stated. Transfer of anhydrous solvents or air-sensitive reagents was accomplished with oven-dried syringes or cannula. Solvents were distilled before use: dichloromethane $\left(\mathrm{CH}_{2} \mathrm{Cl}_{2}\right)$ from calcium hydride, diethyl ether $\left(\mathrm{Et}_{2} \mathrm{O}\right)$ and tetrahydrofuran (THF) from sodium-benzophenone ketyl and toluene from sodium. Commercial reagents and solutions were used as received unless otherwise stated. Thin layer chromatography (TLC) was performed on plates of silica precoated with $0.25 \mathrm{~mm}$ Kieselgel $60 \mathrm{~F}_{254}$ (Merck). Flash columns were packed with 230-400 mesh silica gel (EM Science). Radial chromatography was performed on a Harrison Research Chromatotron Model 7924T with plates of silica precoated with 2 and $4 \mathrm{~mm}$ Silica Gel $60 \mathrm{~F}_{254}$ Containing Gypsum (EM Science). Percentage specifications for mixtures of solvents always refer to the more polar component. Melting points were obtained on a Thomas-Hoover apparatus in open capillary tubes and are uncorrected. Proton nuclear magnetic resonance spectra ( ${ }^{1} \mathrm{H}$ NMR) were recorded on a $300 \mathrm{MHz}$ Varian Unity or $500 \mathrm{MHz}$ Varian VXR instrument. Carbon nuclear magnetic resonance spectra $\left({ }^{13} \mathrm{C} \mathrm{NMR}\right)$ were obtained at $75 \mathrm{MHz}$ (Unity) or at $125 \mathrm{MHz}$ (VXR). Chemical shifts are reported on the $\square$ scale (ppm) and are referenced to deuteriochloroform $\left(\square=7.26 \mathrm{ppm},{ }^{1} \mathrm{H} \mathrm{NMR} ; \square=77.23 \mathrm{ppm},{ }^{13} \mathrm{C} \mathrm{NMR}\right)$ as internal standard. Coupling constants $(J)$ are reported in Hertz $(\mathrm{Hz})$.

Enol triflate 1a. In a $250 \mathrm{~mL}$ round bottom flask was prepared a solution of diisopropylamine $(2.4 \mathrm{~mL}, 17.1 \mathrm{mmol})$ in THF $(70 \mathrm{~mL})$ and the solution was cooled to $-78^{\circ} \mathrm{C}$ before $n$-butyl lithium (2.4 $\mathrm{M}$ in hexanes, $6.7 \mathrm{~mL}, 16 \mathrm{mmol})$ was added via syringe. The resulting mixture was stirred for $30 \mathrm{~min}$ at $-78{ }^{\circ} \mathrm{C}$ before a solution of $2,8,8$ trimethylbicyclo[3.2.1] oct-2-en-7-one $^{1}(2.50 \mathrm{~g}, 15.2 \mathrm{mmol})$ in THF $(30 \mathrm{~mL})$ was added to the LDA solution via cannula over a period of $30 \mathrm{~min}$. The enolate solution was then stirred at -78 ${ }^{\circ} \mathrm{C}$ for $2 \mathrm{~h}$. Solid phenyl trifluoromethanesulfonimide $(5.98 \mathrm{~g}, 16.7 \mathrm{mmol})$ was added and the mixture was allowed to warm to RT overnight. The reaction was quenched by the addition of $\mathrm{H}_{2} \mathrm{O}(100 \mathrm{~mL})$, the layers were separated and the organic layer was washed with $1 \mathrm{~N} \mathrm{NaOH}(2 \mathrm{x}$ $100 \mathrm{~mL})$ and $\mathrm{H}_{2} \mathrm{O}(100 \mathrm{~mL})$. Drying $\left(\mathrm{MgSO}_{4}\right)$, filtration and concentration by rotarary evaporation yielded a brownish oil which was purified by flash chromatography (silica gel, 
hexanes to 5 to $10 \%$ hexanes/EtOAc) to give a colorless oil $(4.07 \mathrm{~g}, 91 \%): \mathrm{R}_{f} 0.62(10 \%$ hexanes/Et $2 \mathrm{O}$ ); IR (thin film) 1637, $1424 \mathrm{~cm}^{-1} ;{ }^{1} \mathrm{H}$ NMR (300 MHz, $\mathrm{CDCl}_{3}$ ) $\square 5.34$ (d, $J=3.4$ $\mathrm{Hz}, 1 \mathrm{H}), 515-5.10(\mathrm{~m}, 1 \mathrm{H}), 2.28-2.16(\mathrm{~m}, 1 \mathrm{H}), 2.16(\mathrm{~s}, 1 \mathrm{H}), 2.14-2.10(\mathrm{~m}, 1 \mathrm{H}), 1.86-1.76(\mathrm{~m}$, 1H), 1.76-1.72 (m, 3H), 1.17 (s, 3H), 1.01 (s, 3H); $\left.{ }^{13} \mathrm{C} \mathrm{NMR} \mathrm{(75} \mathrm{MHz,} \mathrm{CDCl}\right)$ ) 158.3, 137.8, 118.6, 114.5, 54.6, 44.6, 43.8, 26.6, 26.1, 23.7, 20.7 ( $\mathrm{CF}_{3}$ carbon not detected); Anal. Calcd. For $\mathrm{C}_{12} \mathrm{H}_{15} \mathrm{~F}_{3} \mathrm{O}_{3} \mathrm{~S}:$ C, 48.64; H, 5.10. Found: C, 48.73; H, 5.13.

Enol triflate 1b. To a solution of KHMDS (1.22g, $6.15 \mathrm{mmol})$ in THF $(38 \mathrm{~mL})$ cooled to $78{ }^{\circ} \mathrm{C}$ was added dropwise a solution of 7,7-dimethylbicyclo[2.2.1]heptan-2-one ${ }^{1}(0.760 \mathrm{~g}, 5.62$ $\mathrm{mmol})$ in THF $(1 \mathrm{~mL})$ via cannula. The resulting mixture was stirred at $-78{ }^{\circ} \mathrm{C}$ for 1 hour. $\mathrm{N}$ Phenyl trifluoromethanesulfonimide $(2.21 \mathrm{~g}, 6.19 \mathrm{mmol})$ was then added in one portion at -78 ${ }^{\circ} \mathrm{C}$. The mixture was allowed to gradually warm to room temperature. The reaction mixture was quenched with $\mathrm{H}_{2} \mathrm{O}(20 \mathrm{~mL})$ and the organic layer was separated. The aqueous layer was extracted with $\mathrm{Et}_{2} \mathrm{O}(2 \times 50 \mathrm{~mL})$. The combined organic phases were washed with $1 \mathrm{M} \mathrm{KOH}(40$ $\mathrm{mL})$, dried $\left(\mathrm{MgSO}_{4}\right)$, filtered and concentrated. The resulting brown oil was filtered through a plug of silica eluting $20 \%$ EtOAc/hexanes and then purified by radial chromatography ( $4 \mathrm{~mm}$, eluting hexanes $(200 \mathrm{~mL})$ to $10 \%$ EtOAc/hexanes $(100 \mathrm{~mL}))$ to give triflate $\mathbf{1 b}$ as a light tan oil (1.03 g, 72\% yield): $\mathrm{R}_{f} 0.58$ (10\% EtOAc/hexanes); IR (neat) $1420,1214 \mathrm{~cm}^{-1} ;{ }^{1} \mathrm{H}$ NMR (500 $\left.\mathrm{MHz}, \mathrm{CDCl}_{3}\right) \square 5.62(\mathrm{dd}, J=3.3,1.5 \mathrm{~Hz}, 1 \mathrm{H}), 2.43-2.42(\mathrm{~m}, 2 \mathrm{H}), 2.01-1.93(\mathrm{~m}, 2 \mathrm{H}), 1.37-1.31$ (m, 1H), 1.18-1.21 (m, 1H), $1.10(\mathrm{~s}, 3 \mathrm{H}), 0.90(\mathrm{~s}, 3 \mathrm{H}) ;{ }^{13} \mathrm{C}$ NMR $\left(125 \mathrm{MHz}, \mathrm{CDCl}_{3}\right) \square$ 154.8, 119.6, $118.8\left(J_{\mathrm{CF}}=319 \mathrm{~Hz}\right), 56.0,52.3,50.2,25.2,23.8,22.0,22.1$; Anal. Calcd for $\mathrm{C}_{10} \mathrm{H}_{13} \mathrm{~F}_{3} \mathrm{O}_{3} \mathrm{~S}: \mathrm{C}, 44.44 ; \mathrm{H}, 4.95$. Found: $\mathrm{C}, 44.98 ; \mathrm{H}, 4.80$.

Enol triflate 1c. $n$-Butyl lithium $(2.3 \mathrm{M}$ in hexanes, $0.32 \mathrm{~mL}, 0.74 \mathrm{mmol})$ was added to a stirred solution of diisopropylamine $(0.11 \mathrm{~mL}, 0.78 \mathrm{mmol})$ in THF $(5 \mathrm{~mL})$ at $-78{ }^{\circ} \mathrm{C}$. The mixture was allowed to warm to $0{ }^{\circ} \mathrm{C}$, stirred for 15 minutes and cooled $-78{ }^{\circ} \mathrm{C}$. 2,4,4Trimethylbicyclo[3.2.1] oct-2-en-7-one ${ }^{1}(0.115 \mathrm{~g}, 0.70 \mathrm{mmol})$ was added dropwise as a solution in THF $(1 \mathrm{~mL})$ via cannula. The resulting mixture was stirred at $-78^{\circ} \mathrm{C}$ for $1 \mathrm{~h}$ before N-Phenyl trifluoromethanesulfonimide $(0.259 \mathrm{~g}, 0.72 \mathrm{mmol})$ was added in one portion. The mixture was then allowed to gradually warm to room temperature. The reaction mixture was quenched with $\mathrm{H}_{2} \mathrm{O}(5 \mathrm{~mL})$, and the organic layer was separated. The aqueous layer was extracted with $\mathrm{Et}_{2} \mathrm{O}(2$ 
x 10mL). The combined organic phases were dried $\left(\mathrm{MgSO}_{4}\right)$, filtered, and concentrated. The resulting tan oil was purified by flash chromatography (silica gel, 1.7 x $13 \mathrm{~cm}$, eluting hexanes) to yield triflate $1 \mathrm{c}$ as a light tan oil $(0.147 \mathrm{~g}, 70 \%$ yield $): \mathrm{R}_{f} 0.67$ (10\% EtOAc/hexanes); IR (neat) $1425,1211 \mathrm{~cm}^{-1} ;{ }^{1} \mathrm{H}$ NMR $\left(500 \mathrm{MHz}, \mathrm{CDCl}_{3}\right) \square 5.53(\mathrm{~d}, J=3.4 \mathrm{~Hz}, 1 \mathrm{H}), 4.87-4.85$ (m, $1 \mathrm{H}), 2.52(\mathrm{~d}, J=4.4 \mathrm{~Hz}, 1 \mathrm{H}), 2.47-2.45(\mathrm{~m}, 1 \mathrm{H}), 2.20(\mathrm{ddd}, J=9.8,4.9,4.9 \mathrm{~Hz}, 1 \mathrm{H}), 1.95(\mathrm{~d}, J$ $=9.8 \mathrm{~Hz}, 1 \mathrm{H}), 1.76(\mathrm{~d}, J=1.5 \mathrm{~Hz}, 3 \mathrm{H}), 1.11(\mathrm{~s}, 3 \mathrm{H}), 0.89(\mathrm{~s}, 3 \mathrm{H}) ;{ }^{13} \mathrm{C} \mathrm{NMR}\left(125 \mathrm{MHz}, \mathrm{CDCl}_{3}\right)$ $158.8,136.2$, 130.6, $118.8\left(J_{\mathrm{CF}}=319 \mathrm{~Hz}\right), 116.3,47.2,44.5,39.0,34.3,29.9,24.8,23.3$.

Vinylstannane 2c. 1,4-Cyclohexadione monoethylene ketal (4.88 mg, $31.2 \mathrm{mmol})$ was dissolved in THF (390 mL) and trisylhydrazine (11.65 g, $39.0 \mathrm{mmol}$ ) was added in one solid portion. The resulting mixture was stirred for $24 \mathrm{~h}$ at room temperature before it was concentrated under reduced pressure leaving a solid residue which was washed repeatedly with hexanes $(3 \times 100 \mathrm{~mL})$ to give the corresponding hydrazone $(12.95 \mathrm{~g}, 95 \%)$ as a white solid: m.p. 144-145 ${ }^{\circ} \mathrm{C}$ (dec.); $\mathrm{R}_{f} 0.46$ (hexanes/EtOAc 1:1); IR (KBr) 3260, 2958, 1165, $1031 \mathrm{~cm}^{-1} ;{ }^{1} \mathrm{H}$ NMR (500 MHz, $\left.\mathrm{CDCl}_{3}\right) \square 7.27$ (br s, 1H), 7.18 (s, 2H), 4.23 (septet, $\left.J=6.7 \mathrm{~Hz}, 2 \mathrm{H}\right), 4.00$ (s, 4H), 2.91 (septet, $J=7.0 \mathrm{~Hz}, 1 \mathrm{H}), 2.41$ (dd, $J=18.7,6.5 \mathrm{~Hz}, 2 \mathrm{H}), 2.39$ (dd, $J=18.8,7.0 \mathrm{~Hz}$, 2H), $1.79(\mathrm{dd}, J=13.3,6.8 \mathrm{~Hz}, 2 \mathrm{H}), 1.76(\mathrm{dd}, J=13.7,6.8 \mathrm{~Hz}, 2 \mathrm{H}), 1.27(\mathrm{~d}, J=6.8 \mathrm{~Hz}, 12 \mathrm{H})$, $1.26(\mathrm{~d}, J=7.0 \mathrm{~Hz}, 6 \mathrm{H}) ;{ }^{13} \mathrm{C} \mathrm{NMR}\left(125 \mathrm{MHz}, \mathrm{CDCl}_{3}\right) \square 153.4,151.5,131.6,124.0,107.9,64.7$, 34.5, 34.4, 33.2, 32.2, 30.2, 25.0, 23.8, 23.3; Anal. Calcd for $\mathrm{C}_{23} \mathrm{H}_{36} \mathrm{~N}_{2} \mathrm{O}_{4} \mathrm{~S}$ : C, 63.27; H, 8.31. Found: $\mathrm{C}, 63.19 ; \mathrm{H}, 8.36$. In a $25 \mathrm{~mL}$ round-bottom flask equipped with a magnetic stir bar, rubber septum and nitrogen inlet was prepared a solution of the hydrazone (1.71 g, $3.92 \mathrm{mmol})$ in THF/TMEDA ( $8 \mathrm{~mL}, 1: 1)$. To this solution was added n-BuLi (2.3 M solution in hexanes, 3.6 $\mathrm{mL}, 8.2 \mathrm{mmol}$ ) at $-78^{\circ} \mathrm{C}$ and the resulting red solution was allowed to slowly warm to $-10{ }^{\circ} \mathrm{C}$. The now deep purple solution was stirred at $-10^{\circ} \mathrm{C}$ for $30 \mathrm{~min}$, cooled to $-78^{\circ} \mathrm{C}$ and treated with $\mathrm{Me}_{3} \mathrm{SnCl}(1.0 \mathrm{M}$ solution in THF, $5.1 \mathrm{~mL}, 5.1 \mathrm{mmol})$ via syringe to give a green solution which was allowed to warm to room temperature. The reaction was quenched by the addition of $\mathrm{H}_{2} \mathrm{O}(5 \mathrm{~mL})$ and the layers were separated. The organic layer was successively washed with $\mathrm{H}_{2} \mathrm{O}$ (ca. 6 × $20 \mathrm{~mL}$ ) until neutral to litmus, dried $\left(\mathrm{NaSO}_{4}\right)$ and concentrated to leave a yellow residue which was purified by Kugelrohr distillation $\left(0.5 \mathrm{~mm} \mathrm{Hg}, 85-100{ }^{\circ} \mathrm{C}\right)$ furnishing $2 \mathrm{c}$ (831 mg, $70 \%$ ) as a colorless liquid (557 mg, $70 \%$ ): $\mathrm{R}_{f} 0.67$ (50\% EtOAc/hexanes); IR (neat) 2899, 1113, $1031 \mathrm{~cm}^{-1} ;{ }^{1} \mathrm{H}$ NMR (300 MHz, $\left.\mathrm{CDCl}_{3}\right) \square 5.44(\mathrm{tt}, J=3.5,1.9 \mathrm{~Hz}), 3.99$ (s, 4H), 
2.44-2.36 (m, 2H), 2.36-2.32 (m, 2H), $1.75(\mathrm{t}, J=6.4 \mathrm{~Hz}, 2 \mathrm{H}), 0.10(\mathrm{t}, J=27.1 \mathrm{~Hz}, 9 \mathrm{H}) ;{ }^{13} \mathrm{C}$ NMR (75 MHz, $\left.\mathrm{CDCl}_{3}\right) \square$ 140.1, 133.1, 107.7, 64.3 (2C), 37.8, 31.8, 30.3, -10.3; Anal. Calcd. for $\mathrm{C}_{11} \mathrm{H}_{20} \mathrm{O}_{2} \mathrm{Sn}$ : C, 43.61; H, 6.65. Found: $\mathrm{C}, 43.37 ; \mathrm{H}, 6.55$.

Vinylstannane 2d. In a $100 \mathrm{~mL}$ round-bottom flask equipped with a magnetic stir bar, nitrogen inlet and rubber septum was dissolved 5-(trimethylsilyl)-4-pentyn-1-ol ${ }^{2}$ (940 mg, $\left.6.01 \mathrm{mmol}\right)$ in THF $(60 \mathrm{~mL})$. To this solution was added bromo( $\square$-allyl)(bis-acetonitrile)(dicarbonyl)molybdenum ${ }^{3}\left(85 \mathrm{mg}, 0.24 \mathrm{mmol}\right.$ ) in one portion followed by the dropwise addition of $\mathrm{Bu}_{3} \mathrm{SnH}$ $(1.78 \mathrm{~mL}, 6.61 \mathrm{mmol})$ via syringe. The deep red-brown solution was stirred at RT for $30 \mathrm{~min}$ before it was concentrated under reduced pressure furnishing a brown residue which was purified by flash chromatography (silica gel, hexanes/EtOAc 2.5 to 5 to 7.5 to 10 to $12.5 \%$ ) to give vinylstannane $2 \mathrm{~d}$ ( $2.04 \mathrm{~g}, 76 \%$ ) as a colorless liquid: $\mathrm{R}_{f} 0.48$ (hexanes/EtOAc $20 \%$ ); IR (thin film) $2920,1465,1247,855 ;{ }^{1} \mathrm{H}$ NMR $\left(500 \mathrm{MHz}, \mathrm{CDCl}_{3}\right) \square 5.81\left(\mathrm{~s}, J_{\mathrm{SnH}}=49.9 \mathrm{~Hz}, 1 \mathrm{H}\right), 3.63(\mathrm{t}$, $J=6.6 \mathrm{~Hz}, 2 \mathrm{H}), 2.50-2.35(\mathrm{~m}, 2 \mathrm{H}), 1.64-1.59$ (m, 2H), 1.54-1.42 (m, 6H), 1.31 (dtt, $J=7.3,7.3$, $7.3 \mathrm{~Hz}, 6 \mathrm{H}), 1.25(\mathrm{dd}, J=7.2,0.5 \mathrm{~Hz}, 1 \mathrm{H}), 0.90-0.86(\mathrm{~m}, 15 \mathrm{H}), 0.12(\mathrm{~s}, 9 \mathrm{H}) ;{ }^{13} \mathrm{C}$ NMR $(125$ $\left.\mathrm{MHz}, \mathrm{CDCl}_{3}\right) \square$ 169.0, 144.1, 63.3, 37.3, 33.9, 29.3, 27.6, 13.9, 10.2, 0.2; HRMS for $\mathrm{C}_{20} \mathrm{H}_{44} \mathrm{OSiSn}\left(\mathrm{M}^{+}\right)$calcd 448.2183, found 448.2111.

\section{References and Notes}

1. Lightner, D. A.; Bouman, T. D.; Crist, B. V.; Rodgers, S. L.; Knobeloch, M. A.; Jones, A. M. J. Am. Chem. Soc. 1987, 109, 6248.

2. Cochrane, J. S.; Hanson, J. R. J. Chem. Soc., Perkin Trans. 1 1972, 361.

3. Tom Dieck, H.; Friedel, H. J. Organomet. Chem. 1968, 14, 375. 


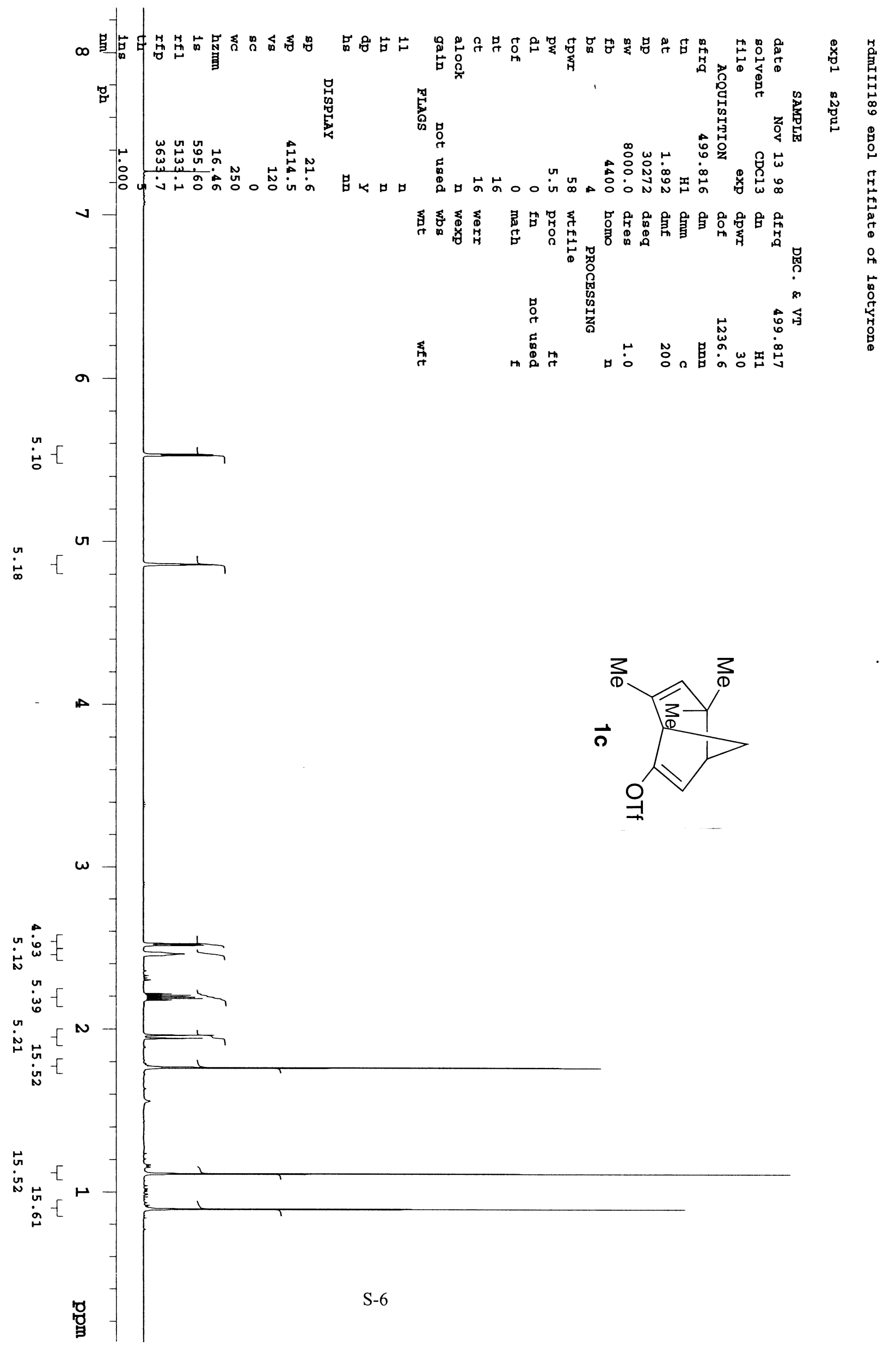




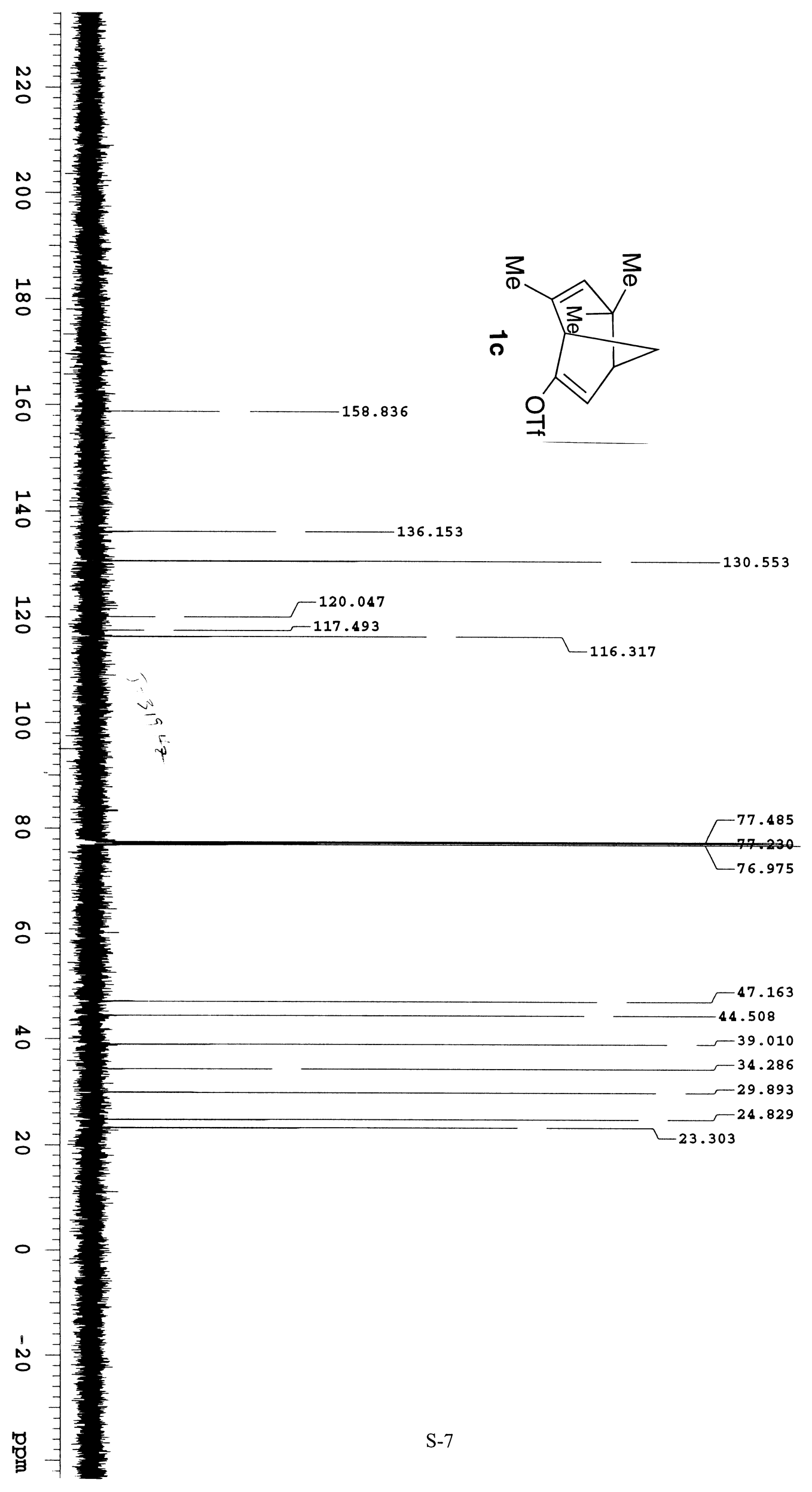




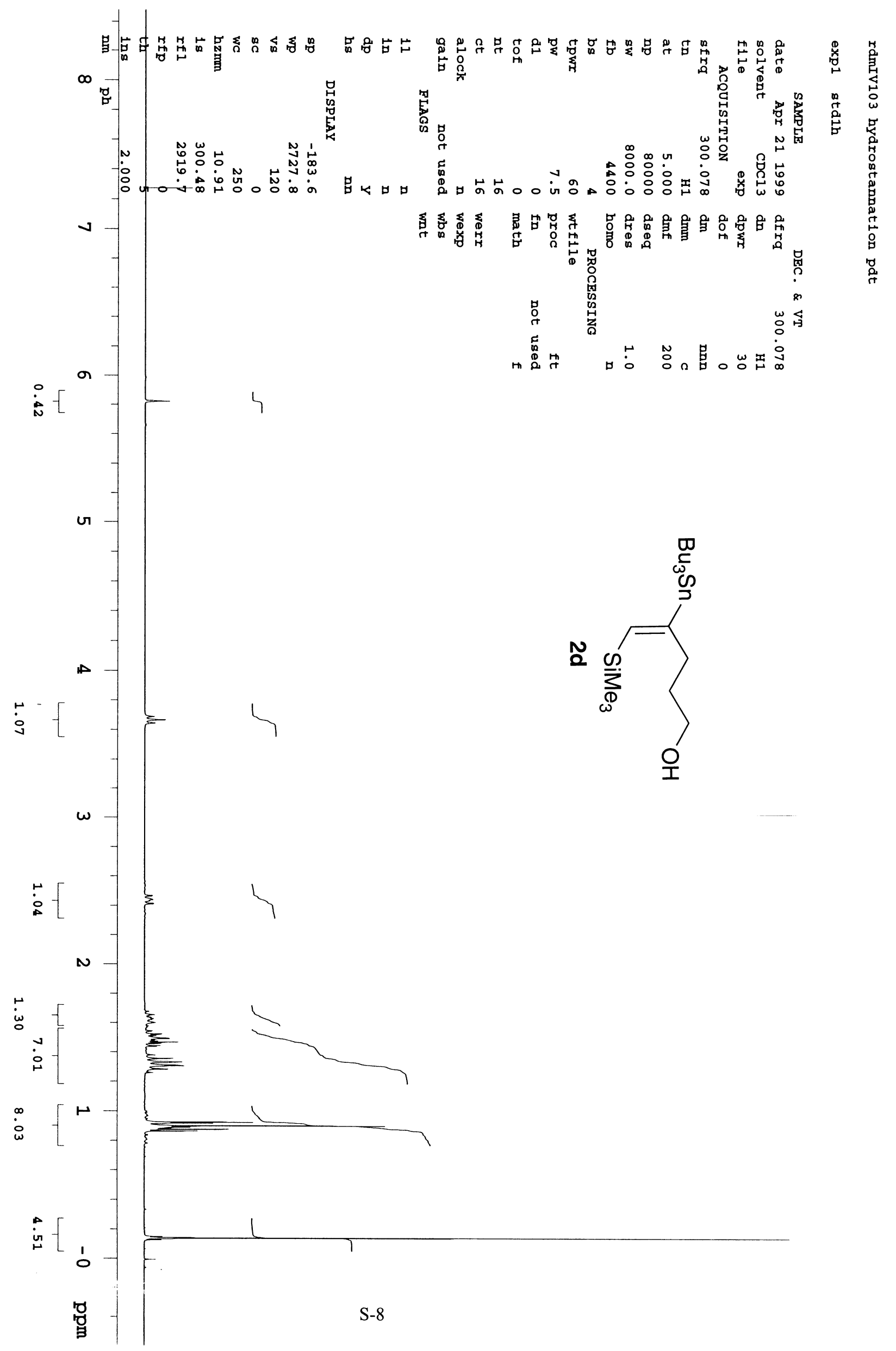




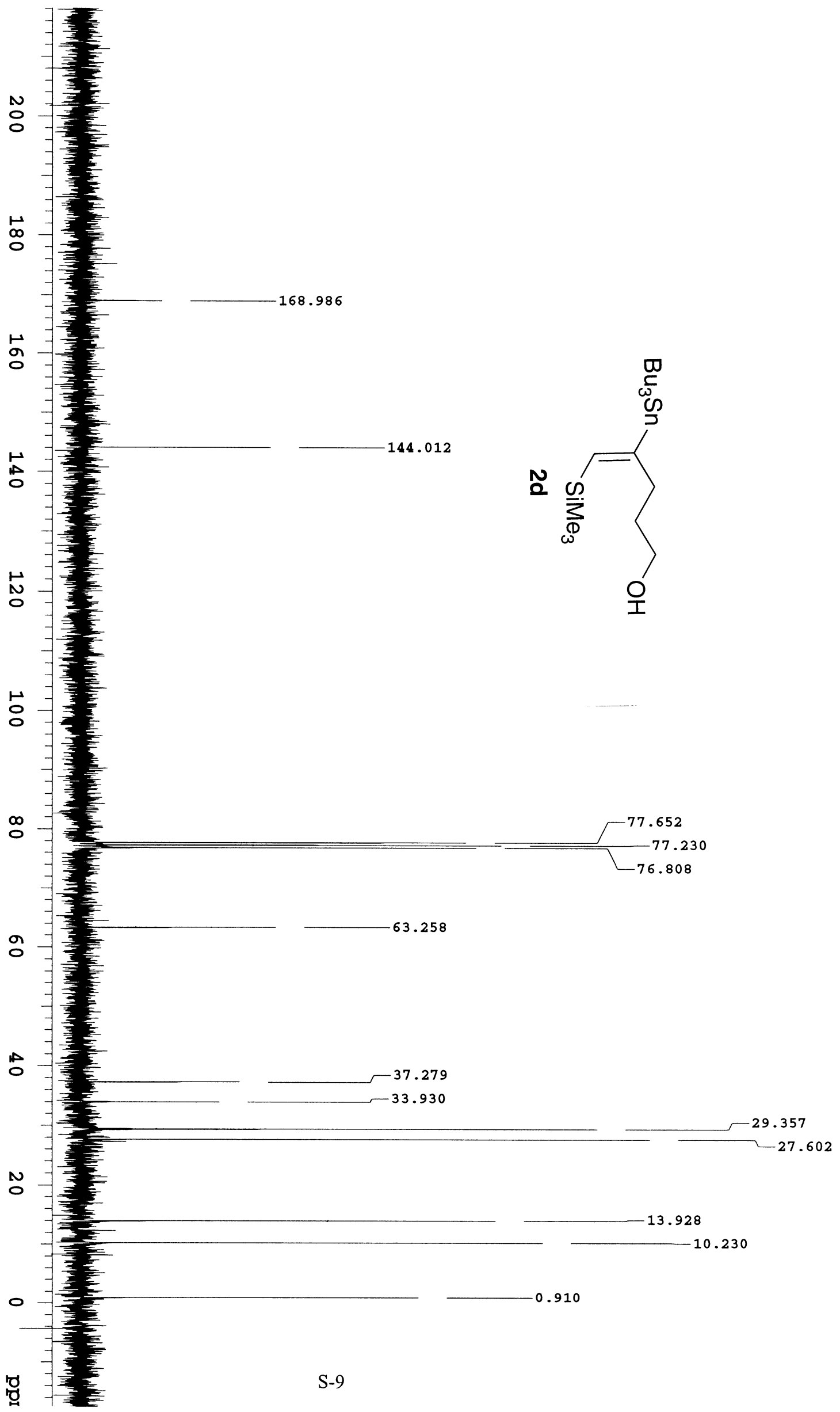




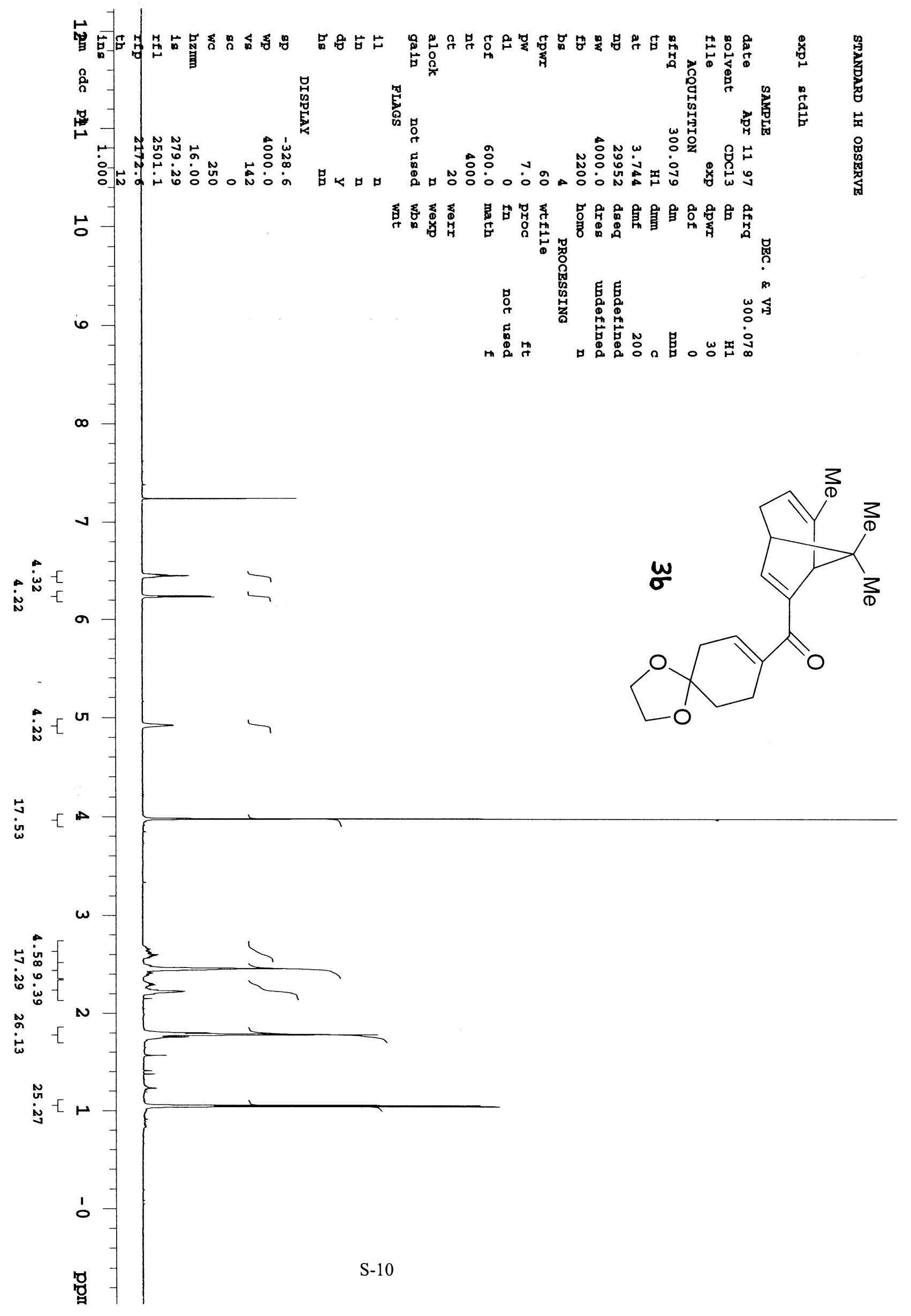




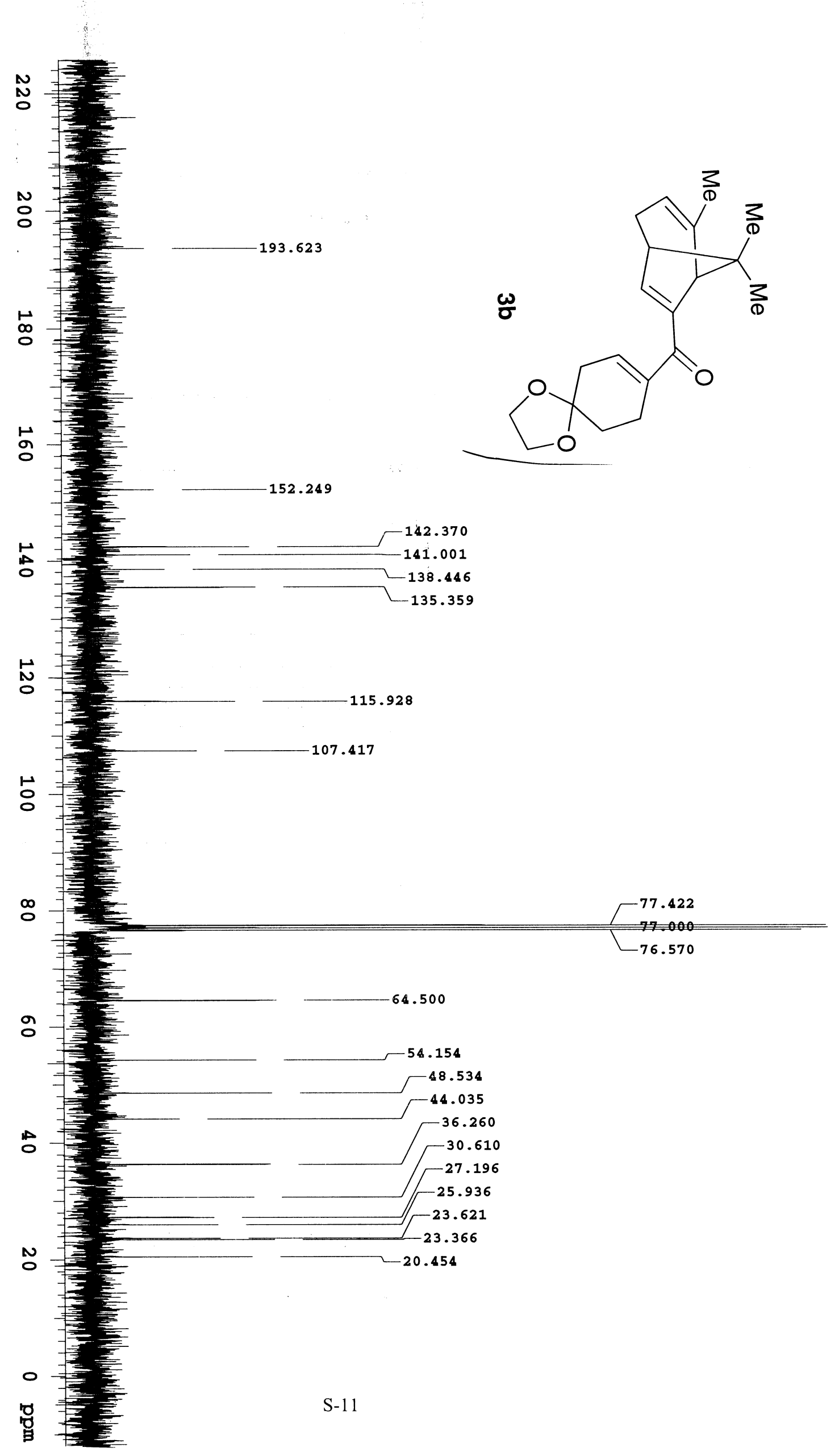




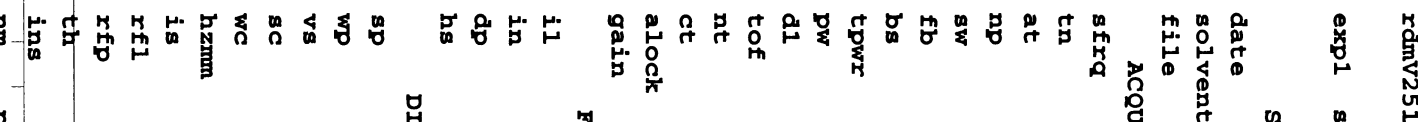
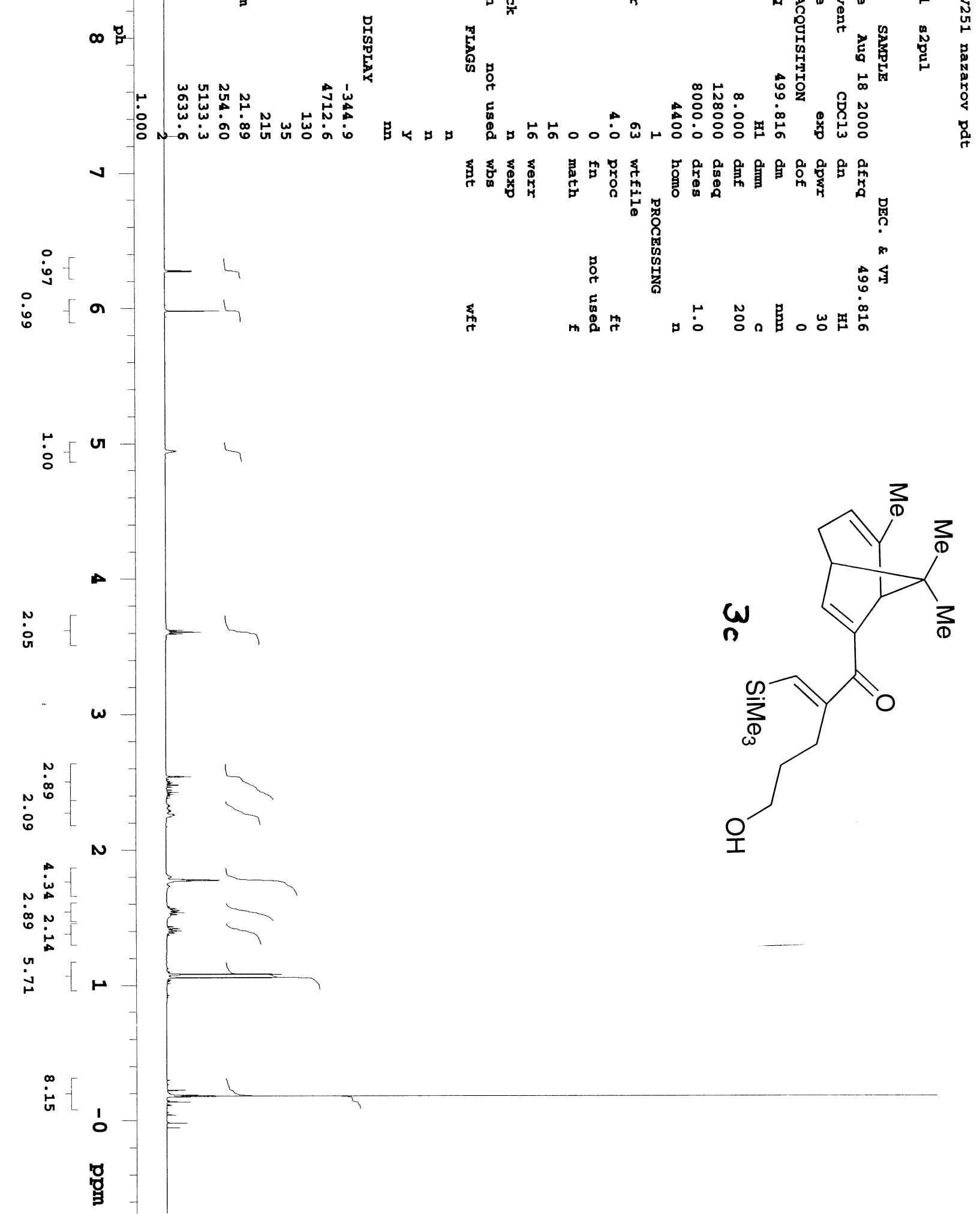


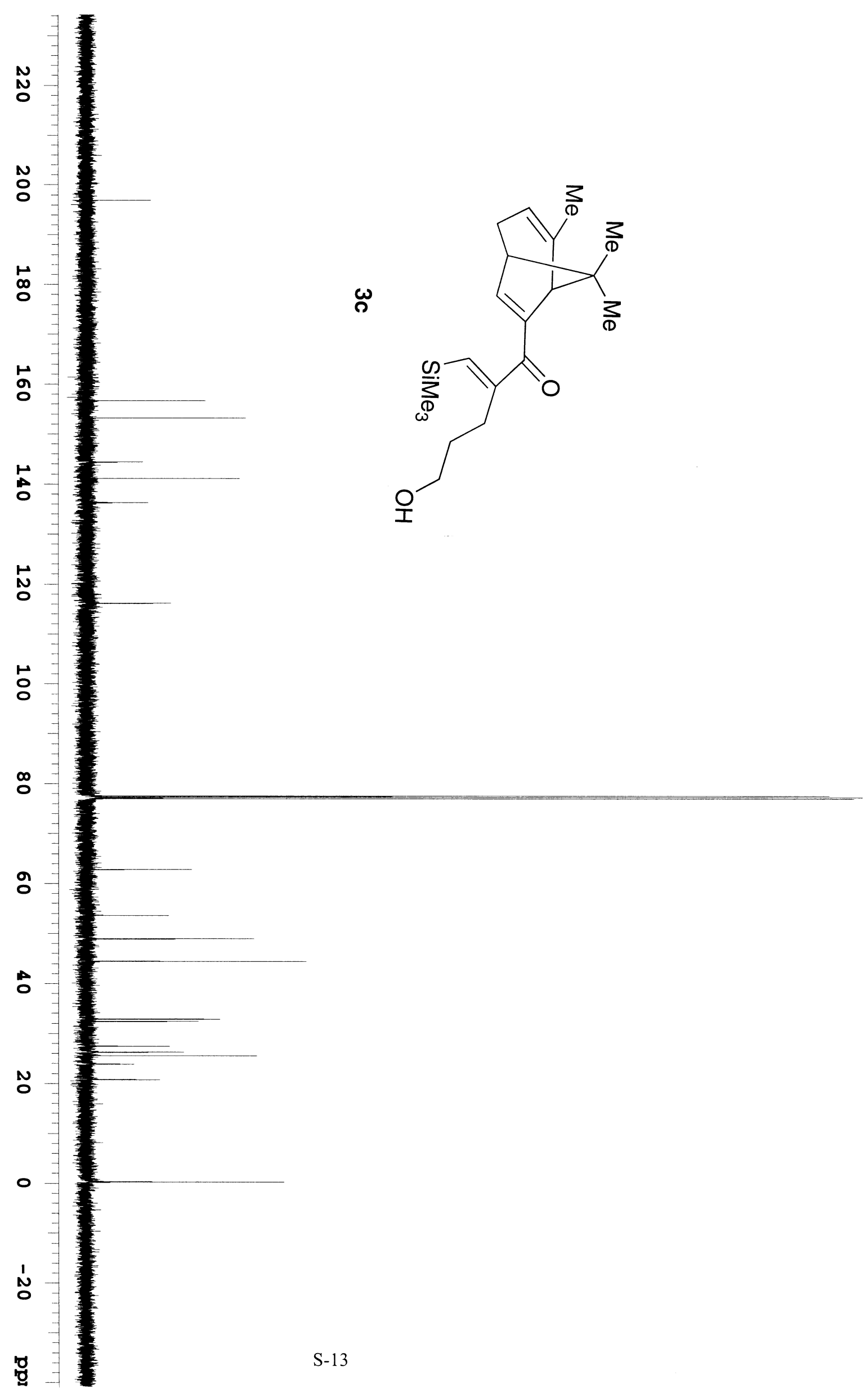




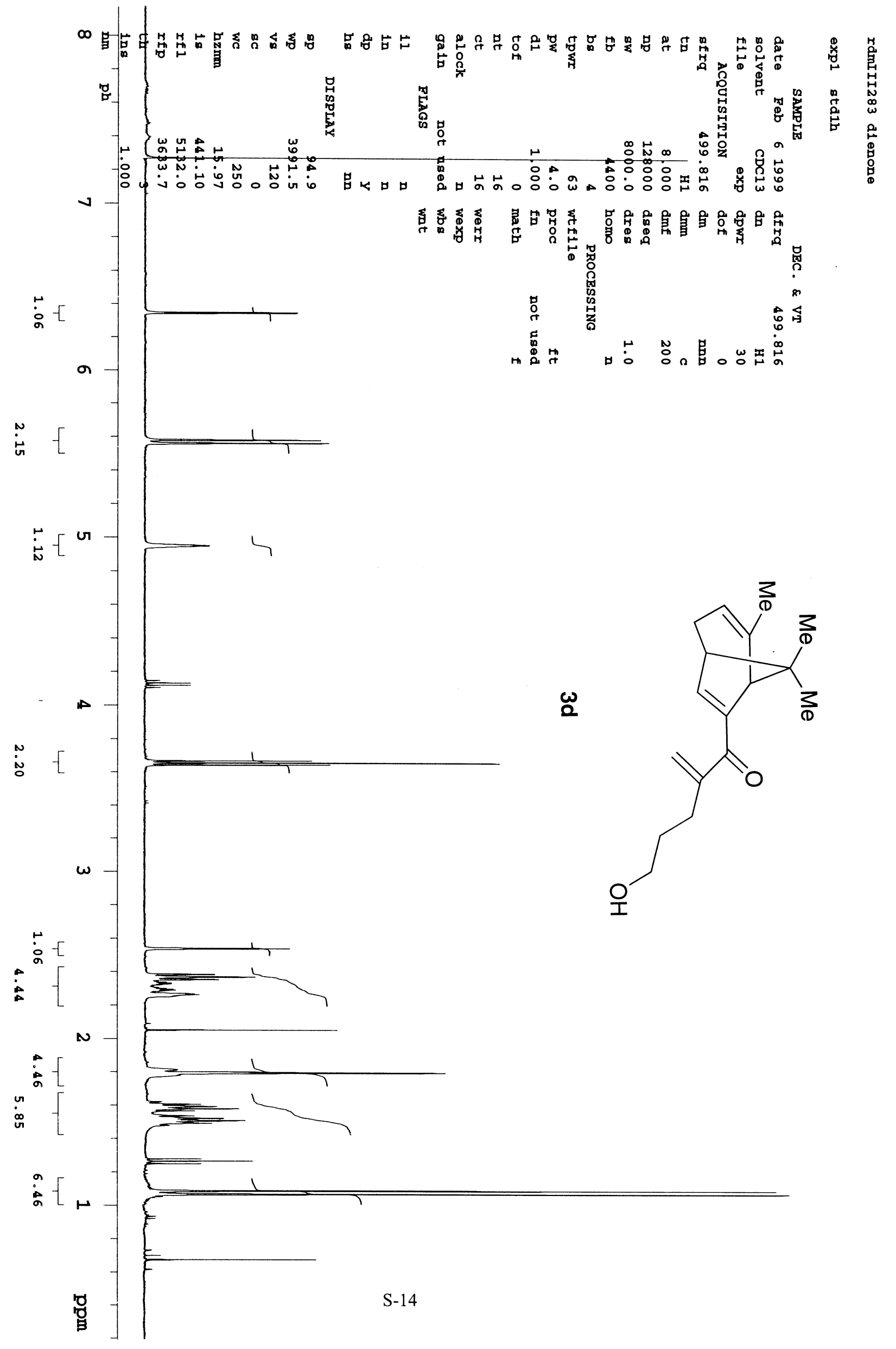




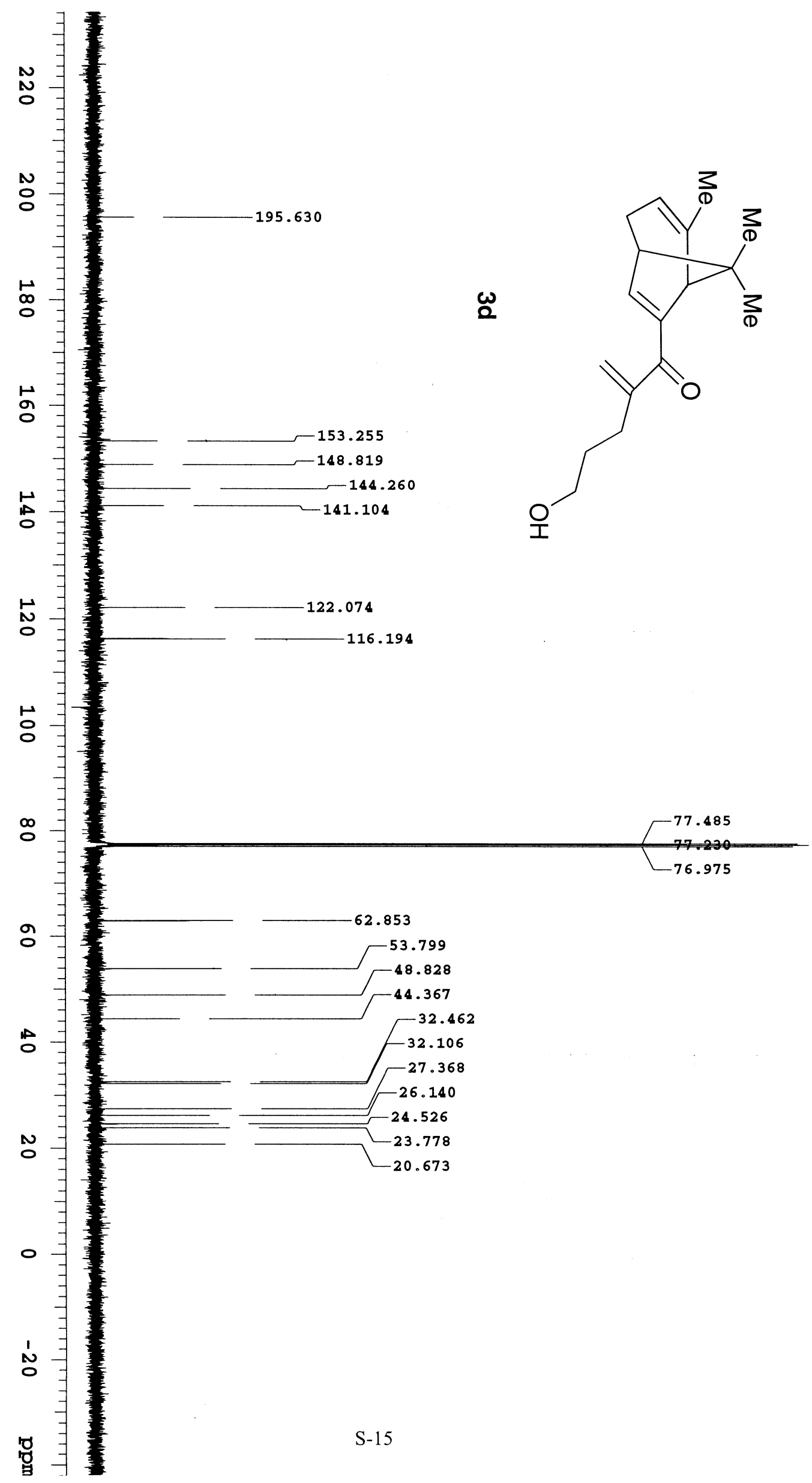




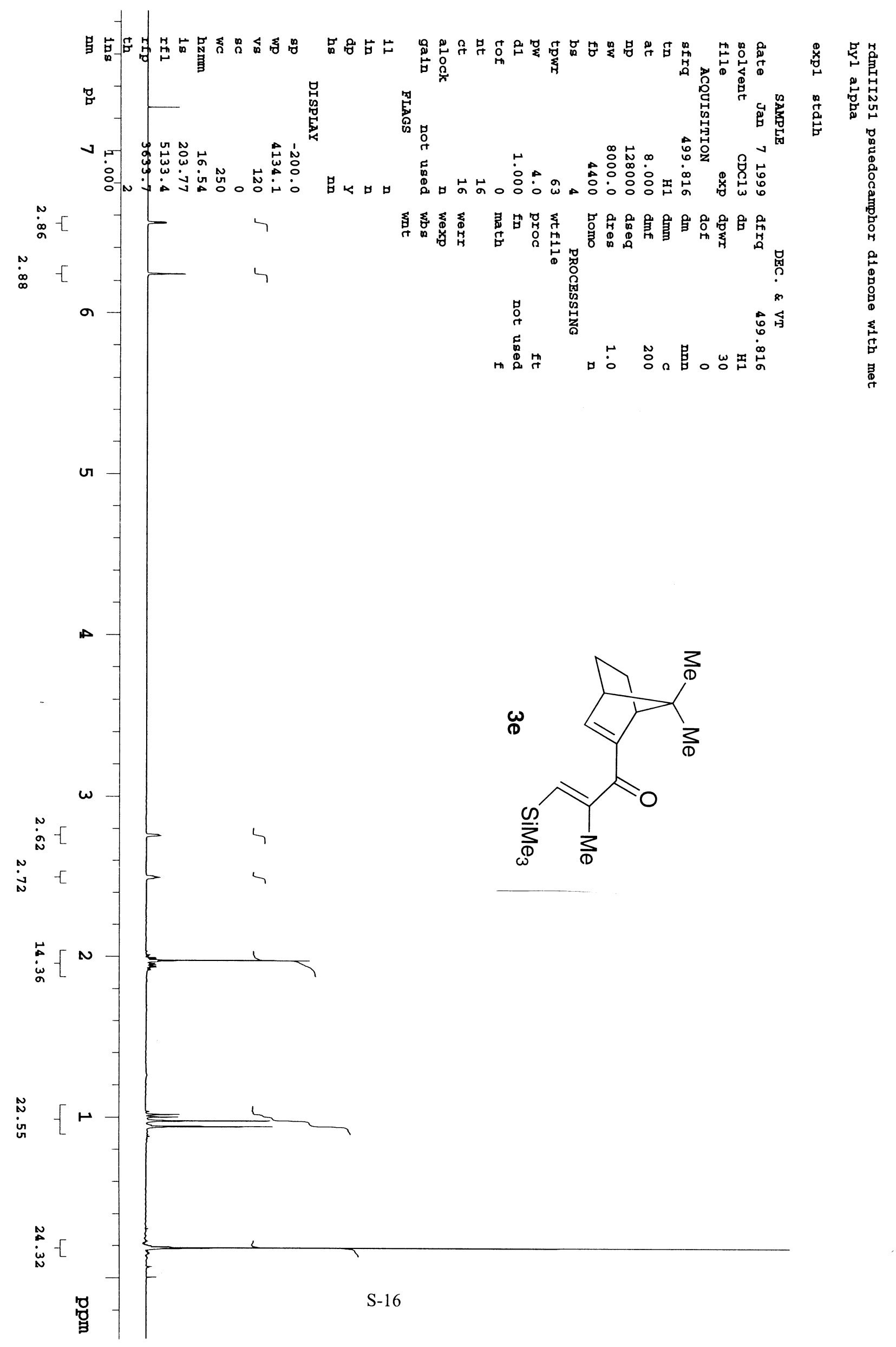




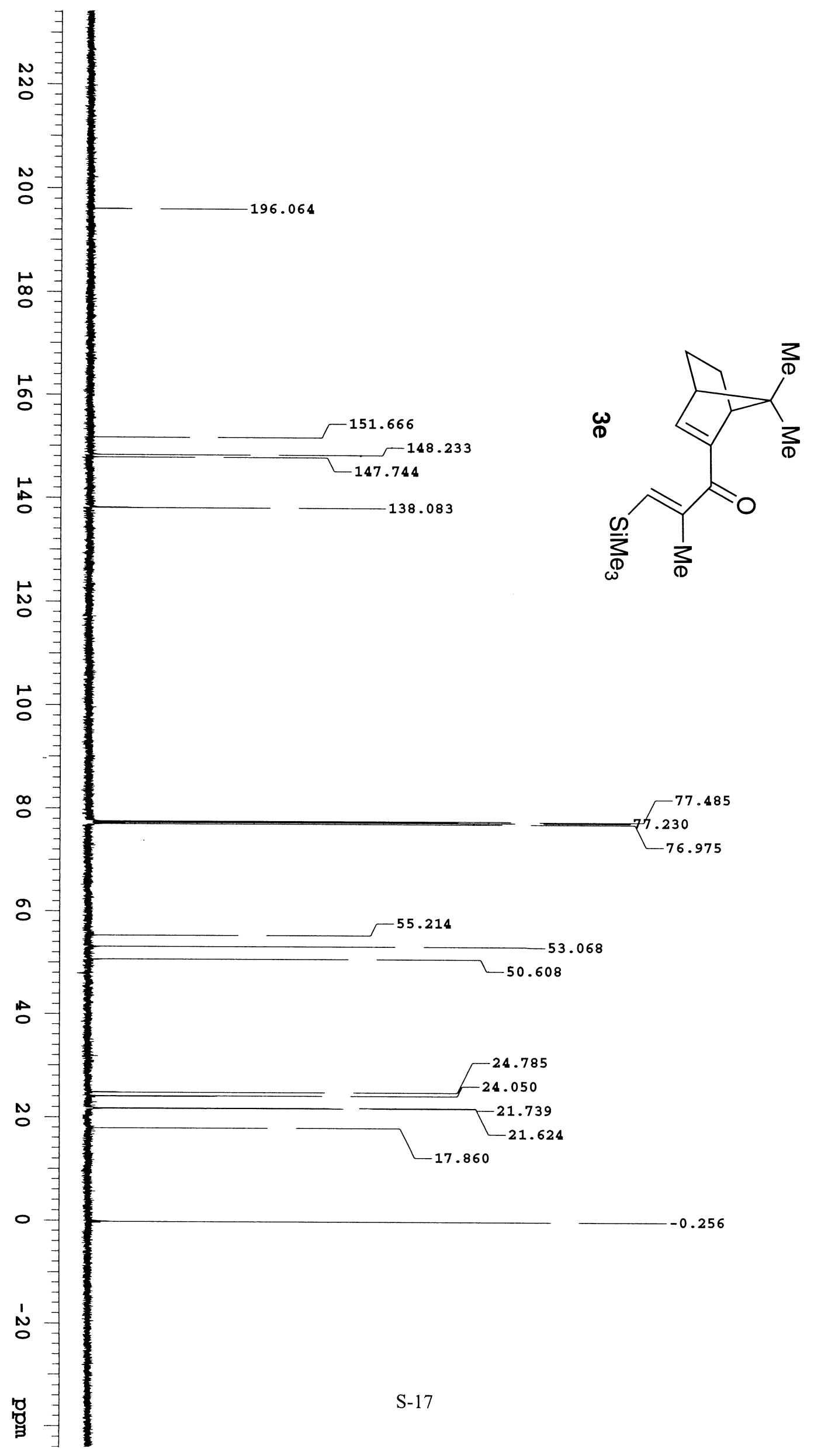




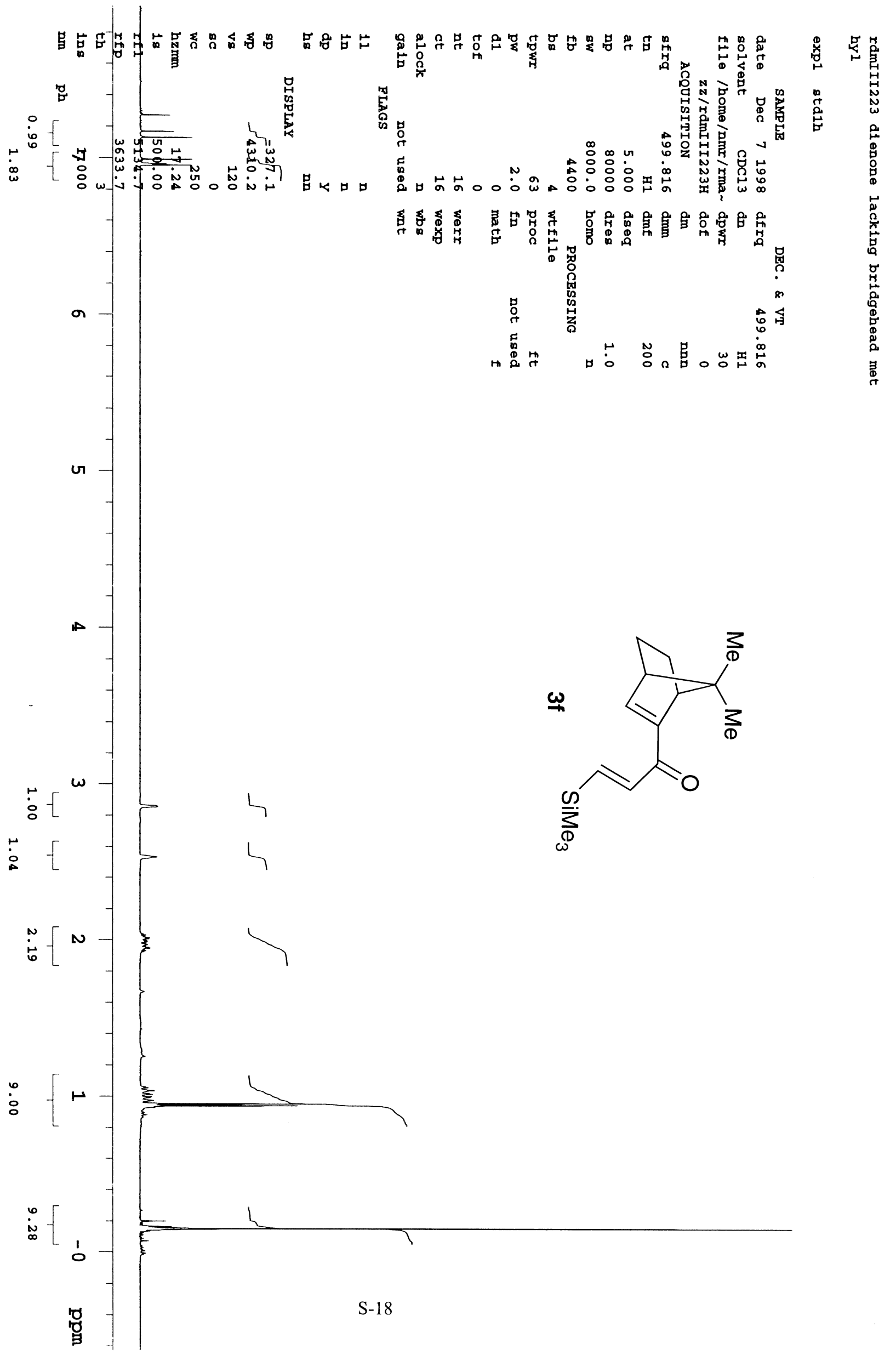




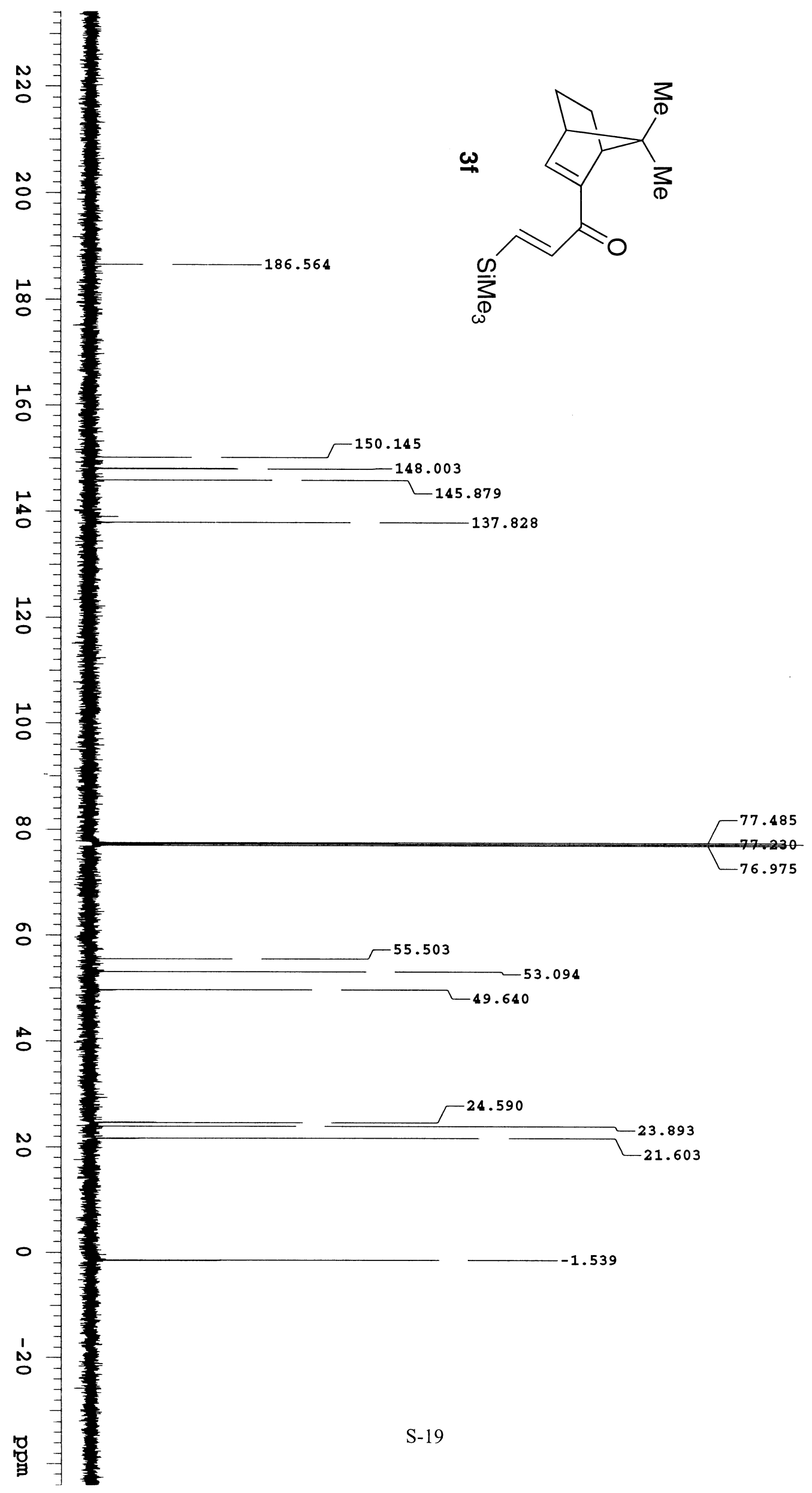




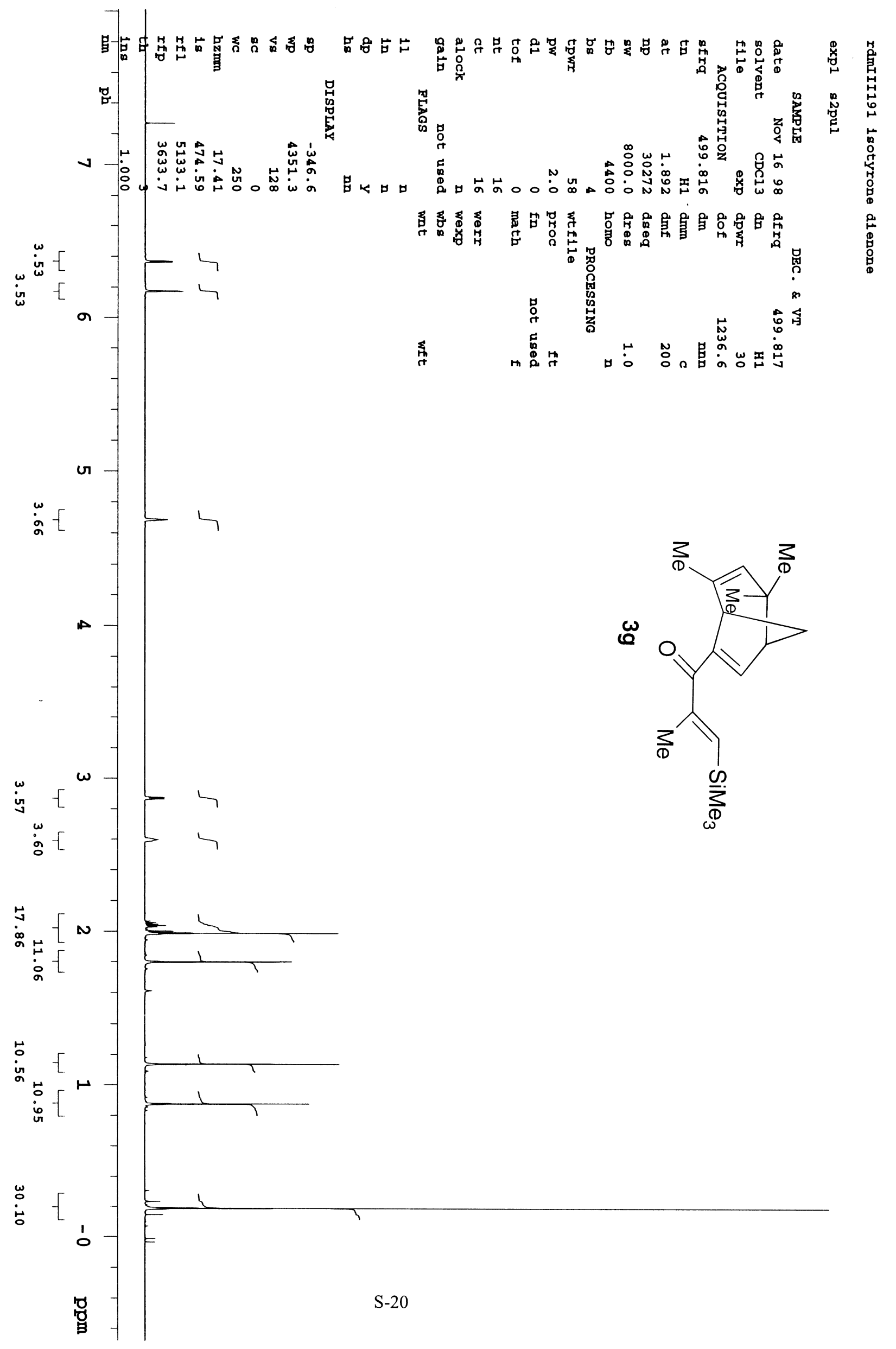




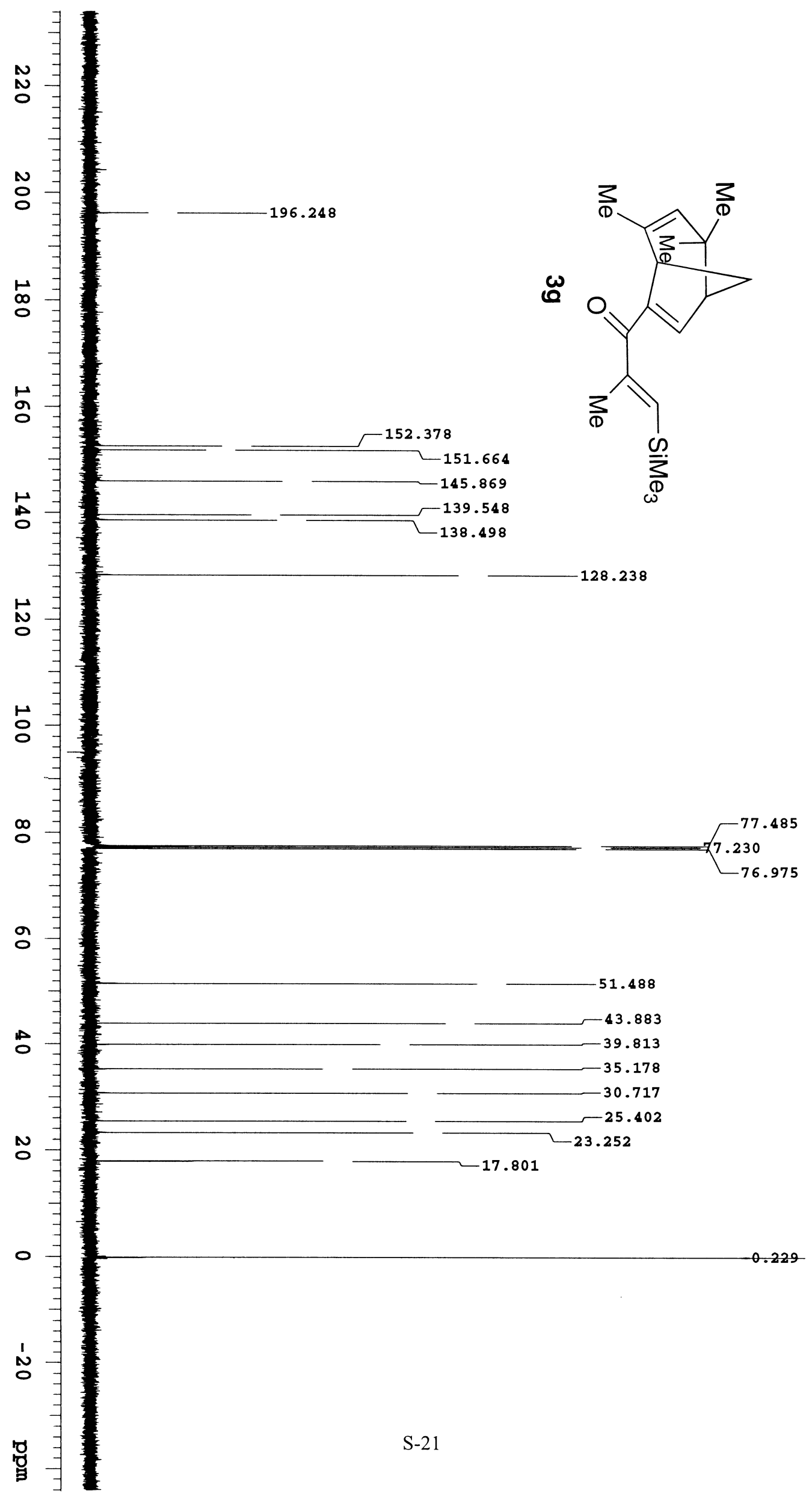




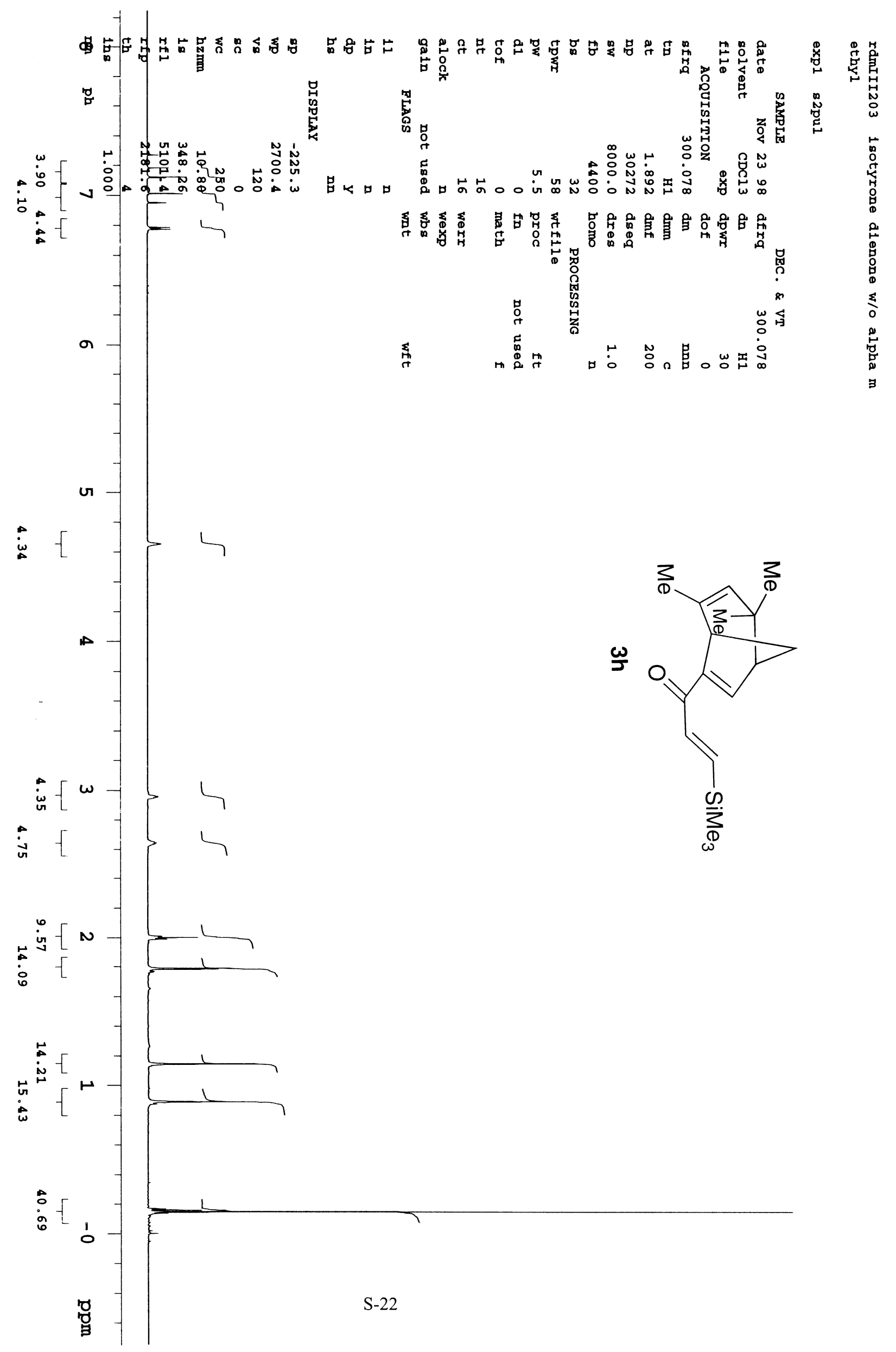




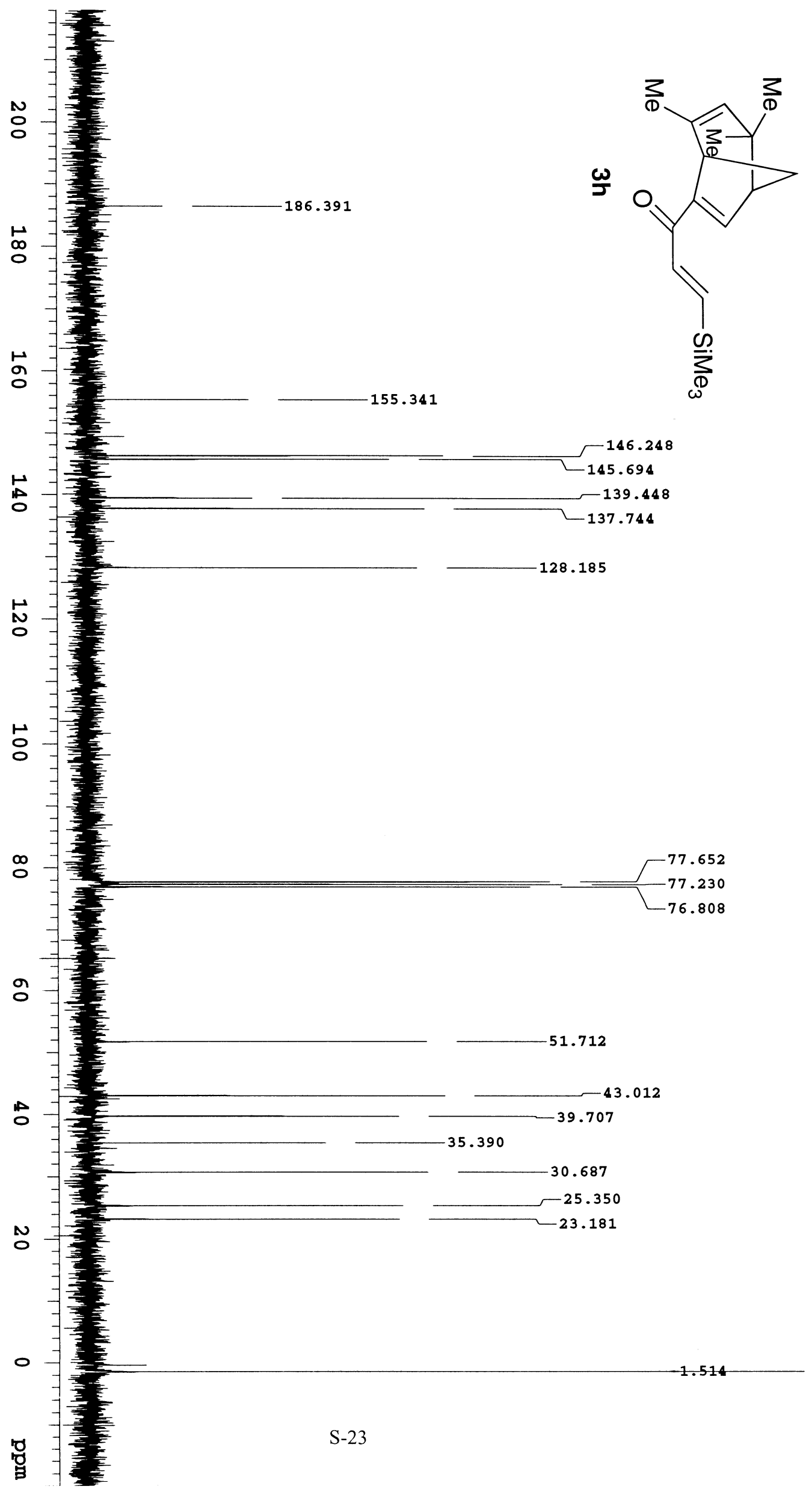



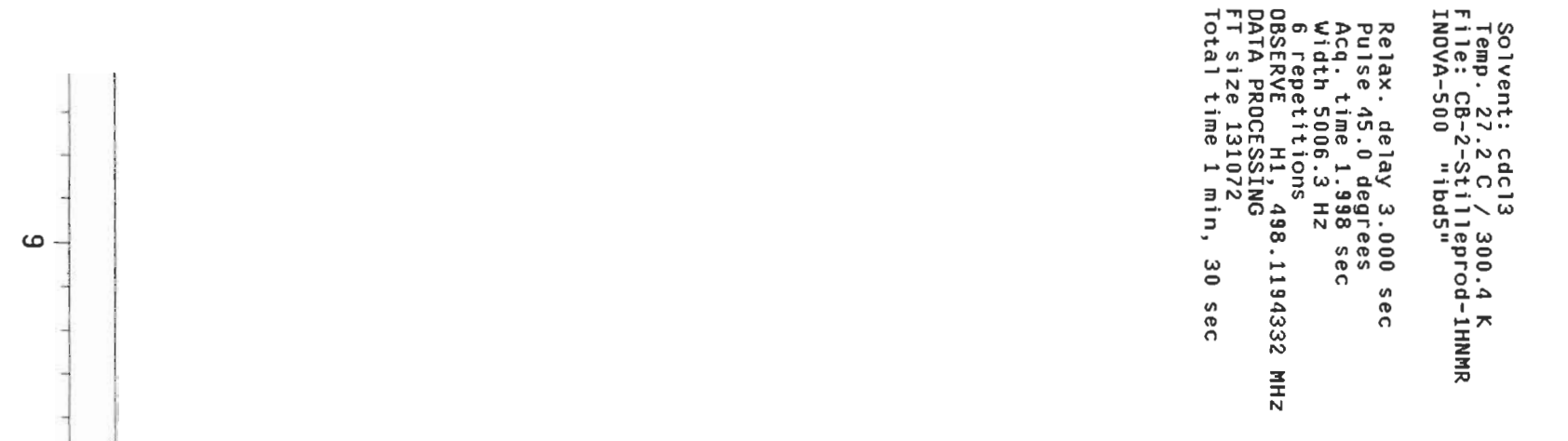

$\infty$

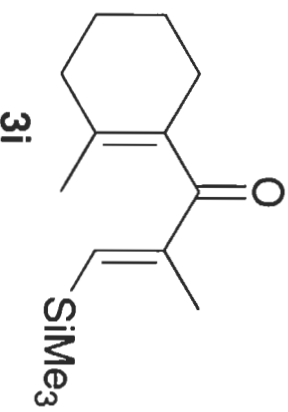

$\omega$
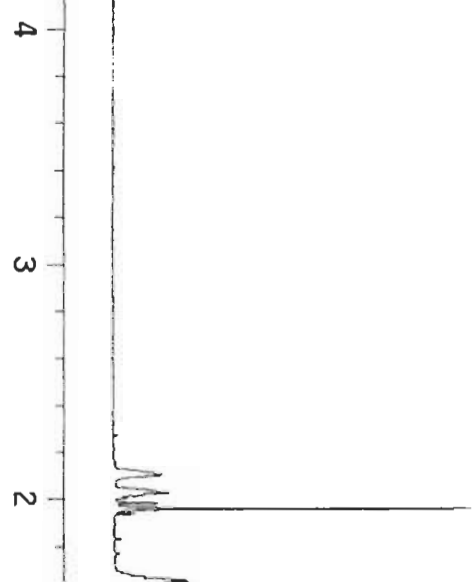


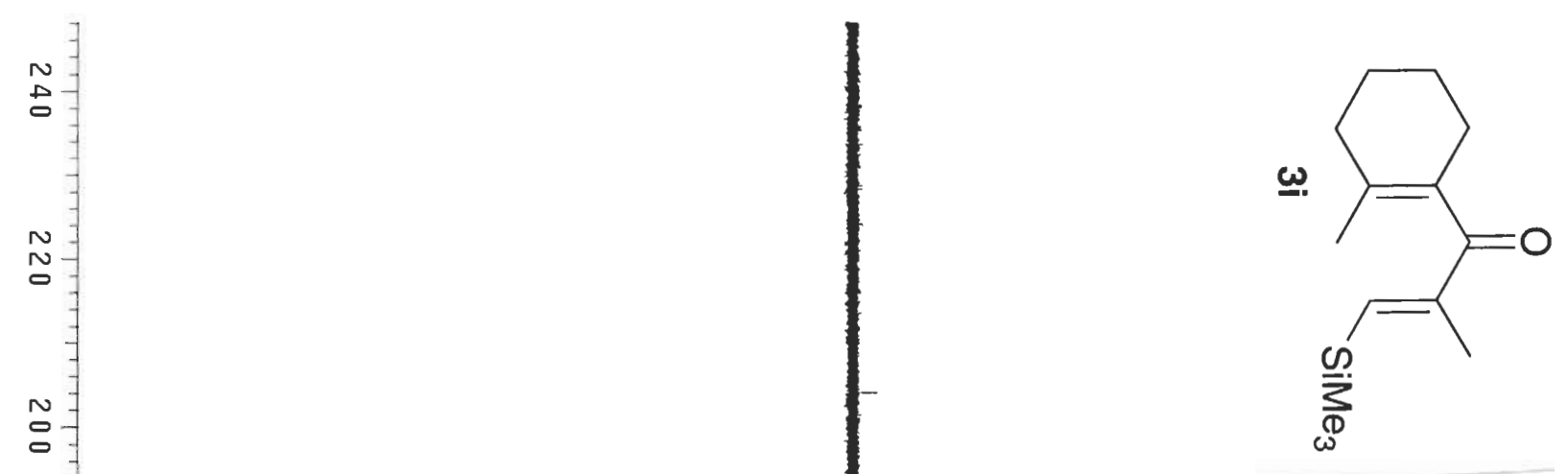

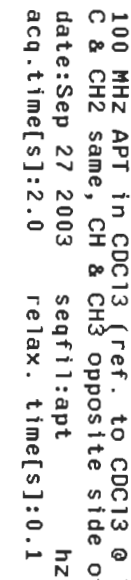

$\stackrel{\infty}{\infty}$

o

D

$\stackrel{N}{0}$

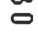

등

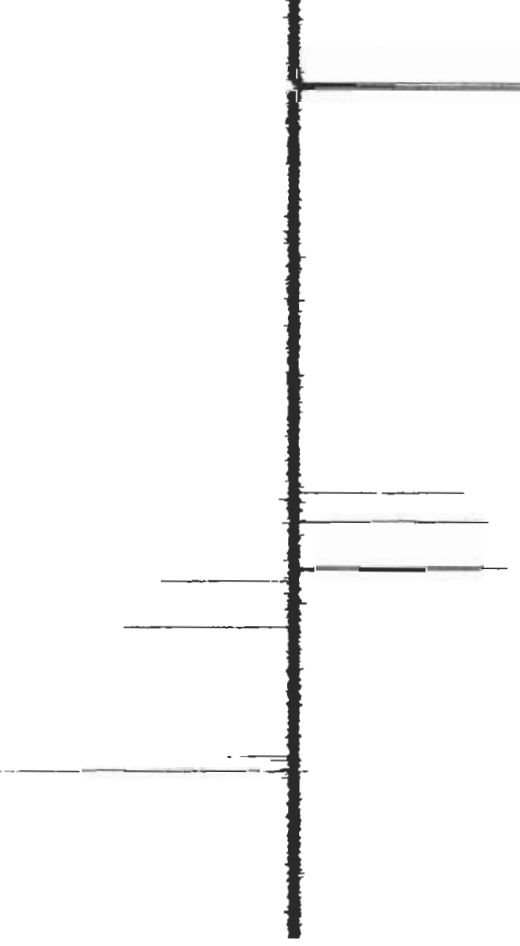

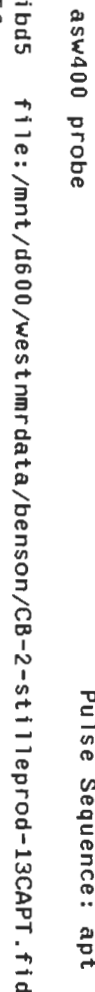

ํㅗㄹ 